\title{
A Language for Behavioural Modelling of Architectural Patterns
}

\author{
Alejandro Sanchez \\ Departamento de Informática \\ Univ. Nacional de San Luis \\ San Luis, Argentina \\ asanchez@unsl.edu.ar
}

\author{
Luis S. Barbosa \\ DI - HASLab \\ Universidade do Minho \\ Braga, Portugal \\ Isb@di.uminho.pt
}

\author{
Daniel Riesco \\ Departamento de Informática \\ Univ. Nacional de San Luis \\ San Luis, Argentina \\ driesco@unsl.edu.ar
}

\begin{abstract}
The complexity of interactions governing the coordination of loosely-coupled services, which forms the core of current software, brought behavioural issues up to the front of architectural concerns. This paper takes such a challenge seriously by lifting typical behaviour modelling techniques to the specification of both types and instances of architectural patterns in which the later ones are connected by ports that behave according to a water flow metaphor. A specific language is introduced for this purpose as well as a translator to $\mathrm{mCRL} 2$ so that the simulation and analysis techniques available in the corresponding toolset can be used to reason about (the behavioural layer of) software architectures. The approach is illustrated in a few examples.
\end{abstract}

\section{Categories and Subject Descriptors}

D.2 [Software Engineering]: Design tools and techniques, Software architectures, Software/program verification

\section{General Terms}

Software architecture

\section{Keywords}

Software architecture, Behaviour modelling, Architectural Pattern

\section{INTRODUCTION}

Continuous evolution towards very large, distributed, heterogeneous, highly dynamic computing systems has shifted both the focus and the method of Software Architecture as a generic design discipline. On the one hand the diversity and complexity of interactions governing the coordination of loosely-coupled services, which forms the core of current software, brought behavioural issues up to the front of architectural concerns. Actually, it is consensual to recognise that the overall structure of software systems [8], whose

Permission to make digital or hard copies of all or part of this work for personal or classroom use is granted without fee provided that copies are not made or distributed for profit or commercial advantage and that copies bear this notice and the full citation on the first page. To copy otherwise, to republish, to post on servers or to redistribute to lists, requires prior specific permission and/or a fee.

BM-FA'11, Jun 06 2011, University of Birmingham, UK Copyright 2011 ACM 978-1-4503-0617-1/11/06 ...\$10.00. study was defined as the specific domain of this discipline in its founding papers, is essentially the structure of their internal interactions and the emergent macro behaviour they entail. On the other hand, informal representations of architectural designs, often relying on graphical notations with poor semantics, are unsuitable for high-assurance software development, precluding any form of rigorous analysis and verification.

In this context the motivation for this paper is twofold: emphasising behaviour modelling in architectural design and doing so in a rigorous, mathematically sound way. Process algebra $[12,4]$, broadly defined as the study of the behaviour of parallel or distributed systems by algebraic means [3], provides a suitable conceptual framework not only to describe software architectures, but also to reason about them either equationally (on top of well studied notions of behavioural equivalence), or through formulation and verification of behavioural requirements expressed in an associated modal logic. Moreover, Process Algebra supports compositional reasoning and abstraction with respect to internal activity of services or components a system is composed of.

This paper introduces a language for specification of architectural patterns, on top of a mature and well-known Process Algebra framework - mCRL2 [10,9]. The language provides mechanisms for defining types as well as instances of them. A type in the language can be defined as a basic architectural element (typically a service/component or connector) with an associated behaviour, or as a set of elements constituting an architectural pattern. In both cases, ports, interaction points that behave according to the intuitive water flow metaphor, can be defined for them. The language also supports hierarchical composition of architectural patterns, allowing the definition of configurations by indifferently attaching ports of pattern or element instances.

A translator of architectural specifications to the mCRL2 modelling language was developed, which brings up a collection of powerful tools for their simulation, graphical visualisations and analysis. mCRL2 is a process algebra, incorporating data and time information, with a number of features which turn it suitable for flexible modelling of behaviour. For example, the introduction of multiactions enables the specification of (not necessarily related) actions that are to be executed together. Most process algebras only allow a single action to be executed atomically thus forcing an order on the execution of actions. They also allow the separation of parallelism and communication: a multiaction simply represents the simultaneous execution of a set of actions, action synchronisation being specified separately. 
After a brief background summary in section 2, the modelling language is introduced in section 3. Section 4 discusses how behavioural analysis of architectural patterns can be performed in this setting. After a presentation of the translation process, analysis in the $\mathrm{mCRL} 2$ framework is illustrated through a number of examples and the basis of a patterns calculus is discussed. Section 5 concludes with a comparison with related work and points some directions for future work.

\section{BACKGROUND}

A language for software design at the architectural level - Architectural Description Language (ADL), must provide a minimum of modelling capabilities and tool support. It is required to allow the explicit specification of both the structural and behavioural dimensions of components, connectors and configurations, and it must also provide tool support to develop and evolve systems in an architecture-based way. A survey describing these requirements in detail can be found in [11].

Among the modelling capabilities, we mention the ability to specify semantics and types for components and connectors - elements, and compositionality for configurations. The semantics of an element is a description of its behaviour in a formal language that enables tool-supported analysis. Then, types allow to define reusable blocks of semantics. Compositionality makes it possible to regard an architecture as a single element in another architectural specification.

An ADL should also include a language feature for the Architectural Pattern concept. The concept is largely applied in the software architecture community and it refers to an architectural abstraction of a design solution to recurring problems. Available catalogs, such as [6] and [15], propose architectural design and evolution by their application. However, its characterisations remain largely informal.

Among the features that tools must provide we mention multiple-views and analysis. The first refers to the possibility of both a textual and a graphical representation. For the second, we focus our attention in the possibility of two checks: refinement - whether an architecture materialising an architectural pattern is a refinement of an element in another pattern (the relevance of this verification is highlighted in [7]); and equivalence - whether an architecture is behaviourally equivalent to another. The mCRL2 language and tool set is a suitable platform to provide these features.

The mCRL2 language [10] is a specification language for describing communication behaviour among systems, which combines a process algebra [4], for the dynamic component, with higher-order abstract equational data types, for the data part. The language is supported by a toolset [9] enabling simulation, visualisation, behavioural reduction and verification. A number of analysis techniques are implemented; their execution resorting, in some cases, to human guidance.

As usual in process algebras, behaviours are described by means of process terms which compose elementary actions (events, communications) through a number of combinators. The reader is referred to [10] for a complete description of mCRL2 and its semantics. For the moment, we limit ourselves to recall the informal meaning of the main process combinators and operators used in the sequel.

Among the combinators of the language we mention: alternative composition $p+q$ - indicates that either of two behaviours $p$ or $q$ can occur; sequential composition $p . q-$ denotes that the process behaves as $p$ and when $p$ finishes it behaves as $q$; parallel composition $p \| q$ - indicates that the actions in $p$ occur independently of actions in $q$; conditional $c \rightarrow p \diamond q-$ represents a process that behaves as $p$ if $c$ holds or as $q$ if it does not; multiactions - denote a set of actions that occur simultaneously; and summand (shown below) indicates a choice among the processes $p(c)$ for $c$ ranging over the possible values in the datatype $D$.

$$
\sum_{c: D} p(c)
$$

Parallel processes can also be influenced by communication operators. We mention four of them: communication, allow, rename, and hide. The communication operator $\Gamma_{c}(p)$ is used to indicate which actions communicate and synchronise within a parallel process expression $p$. The set $C$ contains communication rules of the form $a_{1}|\ldots| a_{n} \rightarrow c$ with $n>0$ and $a_{i}$ and $c$ actions. Each communication rule triggers the replacement of $a_{1}(d)|\ldots| a_{n}(d)$ with $c(d)$ in $p$. The allow operator $\nabla_{V}(p)$ is used to enforce multi-actions in $V$ and block undesired ones. The rename operator $\rho_{R}(p)$, with $R$ a set of renames of the form $a \rightarrow b$, is used to rename action names in $p$. The hide operator $\tau_{I}(p)$ removes action names in the set $I$ from multi-actions and replace them with the invisible action $\tau$.

\section{A LANGUAGE FOR ARCHITECTURAL SPECIFICATIONS}

An architectural specification is built with two basic constructs: pattern and architecture. The first is a formalisation of the concept of architectural pattern and contains elements (connectors and components) showing some behaviour. The second is an instance of a pattern, and describes a particular configuration of (element or pattern) instances. A pattern can be specified without describing any particular architecture. However, in order to be verified, a description of the form Desc needs to contain an architecture and a non empty set of patterns.

$$
\text { Desc }=\langle\operatorname{Set}(\text { Pat }), \text { Arch }\rangle, \text { with patterns }=\pi_{1}
$$

We use projection functions to access tuple components. A projection $\pi_{i}$ allows us to retrieve the $i^{\text {th }}$ tuple component. As a convention, each projection is given a name: the component type name in lower cases. For instance, in (1), $\pi_{2}$ is named $a r c h$. When convenient, we also explicitly name projections as we do in the case of $\pi_{1}$ as patterns.

\subsection{Pattern}

The pattern construct groups descriptions of components and connectors in a single unit, and adopts the form Pat shown in (2). The first component of a tuple pat of this type is a unique identifier and the second is a list that contains its formal parameters. Each $f p$ in $f p a r s(p a t)$ is a tuple with the two components shown in (3): an identifier and an $\mathrm{mCRL} 2$ data type. The example in Listing 1 describes a pattern with Clientserver as id and no parameters. 


$$
\begin{aligned}
\text { Pat }= & \langle\text { Id }, \text { List }(F p), \text { Set }(E)\rangle, \\
& \text { with fpars }=\pi_{2} \wedge \text { elements }=\pi_{3} \\
F p= & \langle I d, \text { DataType }\rangle
\end{aligned}
$$

\section{Listing 1: Client Server Pattern}

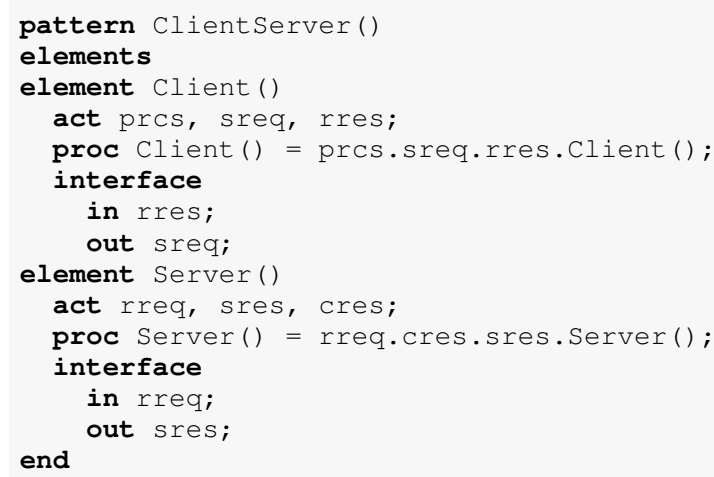

\subsubsection{Element}

The language construct element, allows us to define each element in elements(pat). We use this construct to explicitly characterise both components and connectors. An element $e$ is a tuple that includes, as shown in (4), six components: an identifier, a list of formal parameters $\operatorname{fpars}(e)$; a set $\operatorname{actions}(e)$ of actions; a tuple $\operatorname{mpd}(e)$; a set $\operatorname{procs}(e)$ of process declarations; and a set ports $(e)$ of ports (its interface). Each $f p$ in $f p a r s(e)$ adopts the same form as in (3), and each act in actions $(e)$ the one in (5).

$$
\begin{aligned}
E= & \langle\text { Id }, \text { List }(F p), \text { Set }(\text { Act }), \text { Mpd, Set }(\operatorname{Prc}), \\
& \text { Set }(\text { Prt })\rangle, \text { with fpars }=\pi_{2} \wedge \text { actions }=\pi_{3} \\
& \wedge \text { procs }=\pi_{5} \wedge \text { ports }=\pi_{6} \\
\text { Act }= & \langle\text { Id }, \text { List }(\text { Datatype })\rangle \text { with datatypes }=\pi_{2}
\end{aligned}
$$

The element's behaviour is described using a slightly modified subset of mCRL2. In particular, no init section is considered and process expressions cannot include summands, parallel process combinators, nor communication operators. The content of actions $(e)$ are the actions declared in act sections, and the $\operatorname{mpd}(e)$ and $\operatorname{procs}(e)$ correspond with process declarations in proc sections. The example in Listing 1 shows for each element a section act declaring actions and a section proc defining a process that uses these actions.

The first declared process within the element is the main process declaration (MPD). It constitutes the entry point for its behaviour, and is the one to be started when the element is instantiated. We defer an explanation of what do we mean with "instantiation" until section 4.1, where we describe how instance behaviours are generated. For now, we limit to mention that initial values must be specified for all its formal parameters. The MPD form, shown in (6), has as components: an identifier; a list of formal parameters fpars $(m p d)$; a list ivalues $(m p d)$ of data expressions that match in order and type fpars $(m p d)$, and constitute their initial values; and a process expression body $(m p d)$. The data expressions in ivalues ( $m p d)$ are defined with constants and identifiers declared in fpars $(e)$.

$$
\begin{aligned}
M p d= & \langle I d, \operatorname{List}(F p), \operatorname{List}(I v), \text { Body }\rangle, \\
& \text { with fpars }=\pi_{2} \wedge \text { ivalues }=\pi_{3}
\end{aligned}
$$

Note that the declaration of initial values in formal parameters is not part of mCRL2. Also that sections const, sort, var, eqn, and map can be part of an specification but we do not include tuple components in $e$ for them.

Each proc in $\operatorname{procs}(e)$ is a process declaration that may be invoked from the main process body $\operatorname{body}(\operatorname{mpd}(e))$. Their tuple components are of the form shown in (7), similar to $M p d$, with the difference that they do not have a list of initial values for their formal parameters.

$$
\operatorname{Prc}=\langle I d, \operatorname{List}(F p), \text { Body }\rangle, \text { with fpars }=\pi_{2}
$$

\subsubsection{Interface}

The interface keyword marks the declaration of the set of ports ports $(e)$ that constitute the element's interface (see interfaces for Client and Server respectively starting at lines 6 and 12). We adopt the underlying metaphor of water flow in [2] for ports: an in port receives input from any port connected to it and an out port sends output to all ports connected to it. Ports are synchronous: actually a suitable process algebra expression can be used to emulate any other port behaviour. Each port constitutes a tuple port of the form shown in (8) with a direction (either in or out), and an action as components.

$$
\operatorname{Prt}=\langle\text { Direction }, \text { Act }\rangle
$$

\subsection{Architecture}

An architecture construct describes a configuration that instances adopt. Its declaration is a tuple arch of the form shown in (9). Its tuple components are: an identifier; a pattern reference that must match a declared pattern, i.e., an $i d$ of a tuple pat in patterns(desc); a list apars (arch) of actual parameters that match in order and type fpars (pat); a set of instances instances(arch), a set of attachments atts $(\operatorname{arch})$, and a set of port renames rens(arch). An example architecture is shown below line 15 of Listing 2 .

$$
\begin{aligned}
\text { Arch }= & \langle\text { Id }, \text { Id }, \operatorname{List}(\text { Ap }), \text { Set }(\text { Inst }), \text { Set }(\text { Att }), \\
& \text { Set }(\text { Ren })\rangle, \text { with idPat }=\pi_{2} \wedge \text { apars }=\pi_{3} \\
& \wedge \text { instances }=\pi_{4} \wedge \text { atts }=\pi_{5} \wedge \text { rens }=\pi_{6}
\end{aligned}
$$

Listing 2: Pipe and Filter Architecture

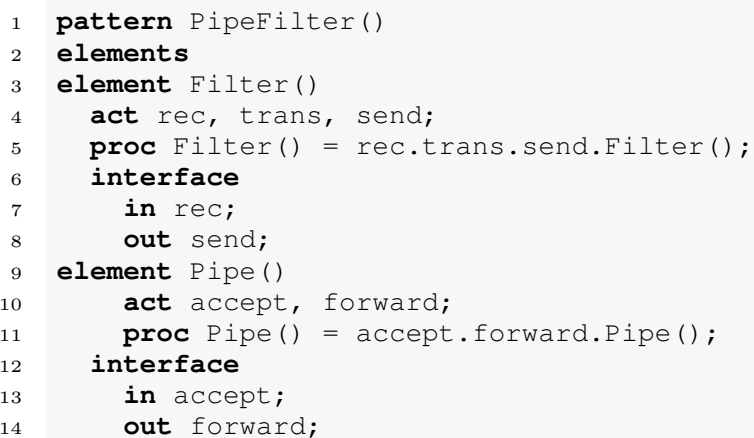




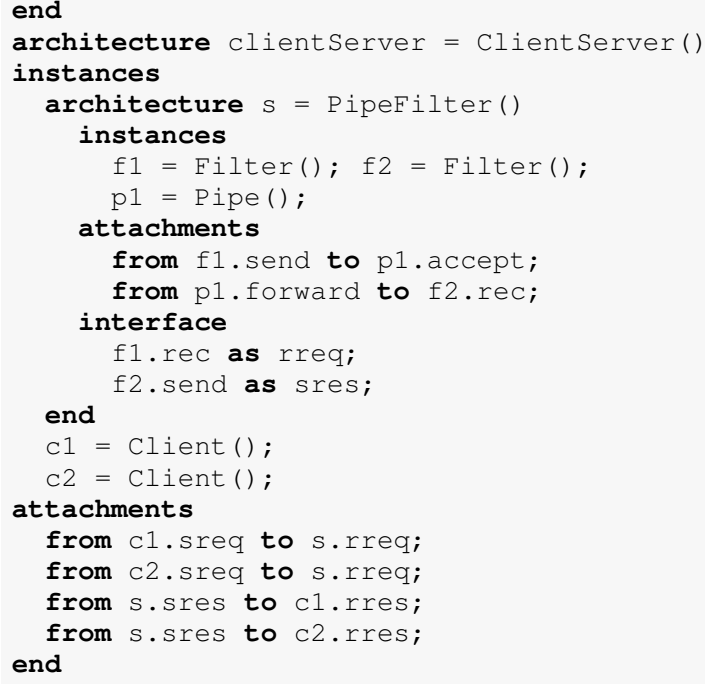

\subsubsection{Instances}

After the keyword instances the set instances(arch) is defined. An instance inst in this set can be either an element instance or an architecture. In the former case, see (10), the instance has an identifier, a reference to an element idElem(inst), and a list apars(inst) of actual parameters that coincide in order and type the element's formal ones $f p a r s(e)$. In the later case, a new architecture construct can be nested as an instance entry. This allows instances to have an architecture as well, building grounds for hierarchically composing specifications. As an example, between lines 18 and 28, an instance is specified as an architecture.

$$
\begin{aligned}
\text { Inst }= & \text { Ainst } \mid \text { Einst } \\
\text { Ainst }= & \text { Arch } \\
\text { Einst }= & \langle\text { Id }, \text { Id }, \operatorname{List}(A p)\rangle \\
& \text { with }\left(\text { idElem }=\pi_{2} \wedge \text { apars }=\pi_{3}\right)
\end{aligned}
$$

\subsubsection{Attachments}

The description of how instances are connected begins after the keyword attachments. Each line in the section defines an attachment, a tuple att in atts (arch) of the form shown in (11). Each attachment includes two port references: one is the out port from(att), and the other the in port to $(a t t)$. They are respectively indicated with the from and to keywords. For instance, the attachment in line 23 indicates that the out port $\mathrm{rec}$ of $\mathrm{f} 1$ communicates with the in port accept of $\mathrm{p} 1$.

$$
A t t=\langle\operatorname{Pr}, \operatorname{Pr}\rangle, \text { with from }=\pi_{1} \wedge t o=\pi_{2}
$$

\subsubsection{Interface}

The architecture's interface is the set rens (arch) of port renames declared upon the keyword interface. Each rename ren contains a port reference $\operatorname{pr}($ ren $)$ and a new name $i d($ ren $)$. Ports not included in rens (arch) are not visible from the outside. Note that including the same port in an attachment and in the interface is incorrect. An example rename is shown in line 26. It indicates that the port rec of $\mathrm{f} 1$ becomes the port $r$ req for the architecture $\mathrm{s}$.
Finally, a port reference (used in (11) and (12)) is a tuple $p r$ adopting one of the two forms shown in (13). The first is used when the reference is to an element instances, and the second when it is to a pattern instances.

$$
\begin{aligned}
\operatorname{Ren} & =\langle\text { Id }, \operatorname{Pr}\rangle \\
\operatorname{Pr} & =\langle\text { Inst }, \operatorname{Prt}\rangle \mid\langle\text { Inst }, \text { Ren }\rangle
\end{aligned}
$$

\section{ARCHITECTURAL ANALYSIS}

\subsection{Generation of $\mathrm{mCRL} 2$ Specifications}

In this section we briefly describe how a process specification is automatically generated from an architectural one. A detailed description can be found in [14].

A generated specification has three main $\mathrm{mCRL} 2$ sections: act, proc and init. We do not mention other sections concerning data expressions since they do not require processing and are just copied. Each of the concatenated functions below formats one of the three sections from a parameter.

$$
\begin{aligned}
& \operatorname{gen}(\text { ainst }: \text { Ainst }) \triangleq \\
& \quad w r t A c t s(\text { acts })+\operatorname{wrtPds}(p d s)+\operatorname{wrtInit}(\operatorname{conf})
\end{aligned}
$$

The first function wrtActs writes the declaration of all actions from a set acts of tuples of the form Act. The second writes the process declarations from a string $p d s$. The third does a similar thing for the initialisation of the system.

We generate the parameters for these functions from an architectural declaration ainst by applying the recursive function genPA. The initial call to this function receives ainst as parameter. Then, it is recursively called for each instance $i$ of ainst and a result $r_{i}$ is obtained. Therefore, we need two versions, one for architectures (15), and one for element instances (16). In both cases the result of its evaluation is a four-component tuple: acts - is the set of actions to be placed in the act section; $p d s$ - is a string containing all process declarations and is to be placed in the proc section; conf - is a string with the configuration the system adopts and is to be placed in the init section; and allows - is a set of strings representing action ids and is used for intermediate calculations during recursive calls. In the next subsections we describe how each of these values is calculated for element instances first and then for pattern instances.

$$
\begin{gathered}
\text { genPA }(\text { ainst }: \text { Ainst }) \triangleq\langle\text { acts }, \text { pds }, \text { conf }, \text { allows }\rangle \\
\forall i \in \text { instances }(\text { ainst }), r_{i}=\text { genPA }(i, \text { ainst }) \\
\text { genPA }(\text { einst }: \text { Einst }) \triangleq\langle\text { acts }, \text { pds }, \text { con } f, \text { allows }\rangle
\end{gathered}
$$

\subsubsection{Generation - Element instance}

The generation of each instance einst of an element $e$ is calculated from the results of processing the bodies of all process declarations defined in $e$ with respect to einst. The function genStep, invoked within genPA for each process body $p$, carries out this processing and returns a tuple containing: $a c t s_{p}$ - the set of actions to be placed in the act section; $p d s_{p}$ - a string with the resulting process expression; allows $s_{p}$ - a set of strings used for intermediate calculations; and hides $s_{p}$ - a set of strings used to build the conf string for the element instance.

We need three rules for genStep. The first is for the alternative composition (17) of two process expressions $p$ and $q$. 
The result is the combination calculated by combine $A C$ of the tuples returned from the recursive call with $p$ and $q$. The function combine $A C$ returns a tuple with the concatenation of the respective $p d s_{p}$, re-assembling the alternative composition expression, and the union of the respective allows $s_{p}$, $a c t s_{p}$ and hides $_{p}$ sets. The second genStep is for conditionals (18). It works in a similar way as the one for alternative composition with the difference that it reassembles a conditional. The third one is for sequential composition (19) and uses another function (20) that processes the individual id.

$$
\begin{aligned}
& \text { genStep }(" \$ p \$+\$ q \$ ", \text { einst }: \text { Einst }) \\
& \quad \triangleq \operatorname{combine} A C(\text { genStep }(p, \text { einst }), \text { genStep }(q, \text { einst })) \\
& \text { genStep }(" \$ c \$ \rightarrow \$ p \$ \diamond \$ q \$ ", \text { einst }: \text { Einst }) \\
& \quad \triangleq \operatorname{combineCond}(c, \text { genStep }(p, \text { einst }), \text { genStep }(q, \text { einst })) \\
& \text { genStep }(" \$ i d \$(\$ x \$) . \$ p \$ ", \text { einst }: \text { Einst }) \\
& \quad \triangleq \operatorname{combineSC}(\text { gen } S t e p(\text { id }, x, \text { einst }), \text { genStep }(p, \text { einst })) \\
& \text { genStep }(i: \text { Id }, x: \text { List }(A p), \text { einst }: \text { Einst }) \\
& \triangleq\left\langle\text { act } s_{p}, p d s_{p}, \text { allows }, \text { hides } s_{p}\right\rangle
\end{aligned}
$$

The genStep for processing an $i d$ requires distinguishing which of the three roles shown below is $i d$ assuming.

- Process Call. In this case $p d s_{p}$ becomes the concatenation of the $i d$ (the process name) with $i d$ (einst) and with its actual parameters if present, and allows $s_{p}$, hides $_{p}$, acts $_{P}$ are empty sets.

- Port. In this case we first need to obtain the list of attached port references attPrts. If the port is locally attached, i.e. at the container architecture ainst, we get it from the set of attachments atts(ainst). If the port is attached at the upper level, and assuming that gparent is the architecture containing ainst, we obtain the set from atts (gparent), after considering the corresponding rename in rens(ainst) (this may need to be repeated). Then we generate the set of actions $a c t s_{p}$ by creating a new action for each $p r$ in attPrts. The identifier for an action derived from a $p r$ is the concatenation of $i d$ with $i d($ einst) and $i d($ inst $(p r))$. Since ports behave differently according their direction, we need to generate the process expression $p d s_{p}$ according the cases as follows:

- In. The process expression is the alternative composition of all actions in $a c t s_{p}$. When an action receives parameters, we also need to surround the expression with a summand as the one shown below for each parameter $d$ of datatype $D$ not bound to any value.

$$
\sum_{d: D} p d s_{p}
$$

- Out. The process expression is the alternative composition of the multiactions generated from the actions in each set of the power set $P\left(a c t s_{p}\right)$ (the empty set must be removed first). An example of the generated expression is shown in Listing 3 , for the port in line 27 of Listing 2 .

The allows $s_{p}$ is the set of the action ids taken from $a c t s_{p}$, and hides $_{p}$ is the empty set.
- Internal Action or Unattached Port. Then $p d s_{p}$ is an action named as the concatenation of $i d$ with $i d$ (einst). The $a c t s_{p}$ is a set with this action, and allows $s_{p}$ and hides $_{p}$ are sets containing only the generated id.

\section{Listing 3: Out Port Example}

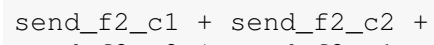

Note that the tactic for generating identifiers is a naive solution in the sense that it ensures different ones when it may not be necessary. An optimised solution aiming at the minimisation of the LTS state number is part of future work.

Once we obtained a result (containing act $s_{p}, p d s_{p}, a l l o w s_{p}$, and hides $_{p}$ ) from each process body, we combine them. With the exception of the body corresponding to the main process, all string expressions $p d s_{p}$ are concatenated into $p d s$ (previously adding their process headers). The sets (allow $s_{p}$, acts $s_{p}$ and hides $_{p}$ ) are position-wise combined by applying set union to become the sets allows, acts and hides.

Subsequently, we generate the conf string for the element instance. For this, we concatenate the element name $i d(e)$, with the instance name $i d($ einst $)$, and the actual parameters apars(einst). For example, the conf generated from the line 29 in Listing 2 is Client_c1. In the case that the element instance has actual parameters, they are appended after the instance id; e.g. $c 1=C$ lient(3) becomes Client_c1(3). In the case that we want to carry out behavioural comparisons and the set hides is not empty, we generate another expression as shown in (21).

$$
\operatorname{con} f_{h}=\tau_{\text {hides }} \operatorname{conf}
$$

We still need to concatenate to the $p d s$ the MPD $\operatorname{mpd}(e)$ In order to use the initialisation values ivalues $(\operatorname{mpd}(e))$ two process declarations are required. The example MPD in Listing 4 and the generated code in Listing 5 illustrate how it is done. Subsequently, the process declaration for the MPD can be concatenated to $p d s$.

\section{Listing 4: Example MPD}

element Client (idC:Nat)

proc

Client $($ id: Nat $=$ idC $)=\ldots$

\section{Listing 5: Example MPD - Generated mCRL2}

1 Client_c1 (idC:Nat) = Client_mp_c1 (idC);

2 Client_mp_c1(id:Nat) $=\ldots$

\subsubsection{Generation - Pattern Instance}

The result of evaluating genPA with an architecture ainst is a tuple $r$ of the form $\langle a c t s, p d s$, conf, allows $\rangle$ as shown in (15). The tuple components in $r$ are generated upon the set of results $r_{i}$ of recursively applying genPA with each instance $i$ in instances(ainst).

The tuple component conf in the result is a string with the $\mathrm{mCRL} 2$ operators $\rho, \nabla$, and $\Gamma$, nested in that order. The communication operator $\Gamma$ takes $C$ as the set of communications and the parallel combination of the $\pi_{3}$ of each $r_{i}$ (which is the respective conf $f_{i}$ of each instance $i$ ). The allow operator $\nabla$, taking as parameter the set $V$ of action ids, is used 
to enforce communications and to rule out undesired action combinations. The rename operator $\rho$ is applied then with the set $R$ of renamings. The $\operatorname{con}_{h}$ string is calculated as the hiding operator $\tau$ applied to con $f$ taking $H$ as argument. As we will mention in Section 4.2, it is used when specifications are generated to carry out behavioural comparisons.

$$
\begin{aligned}
& \operatorname{conf}=\rho_{R}\left(\nabla_{V}\left(\Gamma_{C}\left(\prod_{\text {ei } \in \text { insts }(\text { ainst })} \pi_{3}\left(r_{i}\right)\right)\right)\right) \\
& \operatorname{con} f_{h}=\tau_{H}(\text { conf })
\end{aligned}
$$

Each rule in the set $C$ of communication rules is generated from each attachment in atts(ainst). Identifiers are concatenated as it is shown in example (24), which is the generated rule for the attachment in line 32 of Listing 2 .

$$
c r=\left\langle s r e q \_c 1 \_s, r r e s \_s \_c 1, s r e q \_c 1 \_r r e s \_s\right\rangle
$$

The calculation of the set $R$ of rename rules requires more work. For each ren in rens(ainst) we need the set attPrts of attached port references in the upper level. Then, for each prt in attPrts we generate a rename rule $r r$ as shown in (25). An example of the generated rename rules for the lines 26 and 27 in Listing 2 is shown in Listing 6

$$
\begin{aligned}
& r r=\left\langle i d_{\text {old }}, i d_{\text {new }}\right\rangle \\
& i d_{\text {old }}=i d(\operatorname{pr}(\text { ren }))+i d(\operatorname{inst}(\operatorname{pr}(\text { ren })))+i d(\operatorname{inst}(\text { prt })) \\
& i d_{\text {new }}=i d(\text { ren })+i d(\operatorname{inst}(\operatorname{prt}))+i d(\operatorname{inst}(\operatorname{pr}(\text { ren })))
\end{aligned}
$$

\section{Listing 6: Rename Rules - Generated mCRL2}

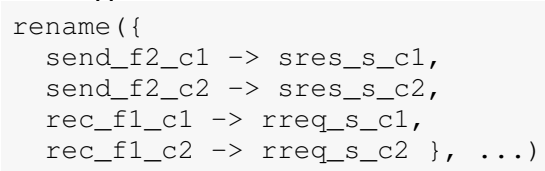

The set $V$ for the allow operator is calculated as the union of three sets: the set of action ids resulting from the projection $\pi_{3}(c r)$ for each synchronisation rule $c r$ in $C$; the set of action ids resulting from the projection $\pi_{1}(r r)$ of each rename rule in $R$; the set resulting from the union of each set $\pi_{4}\left(r_{i}\right)$, i.e., the allows $s_{i}$ set obtained from each instance $i$ of the architecture.

The set $H$ for the hiding operator contains all ids of synchronisation actions in $C$, i.e. $\left\{\right.$ sid $\mid$ sid $\left.=\pi_{3}(c r) \forall c r \in C\right\}$.

The last tuple component of $r$, acts, is the union of the three sets as follows: the set of actions resulting from the projection $\pi_{3}(\mathrm{cr})$ of each $\mathrm{cr}$ in $C$; the set of actions resulting from the projection $\pi_{1}(r r)$ of each $r r$ in $R$; and the set resulting from the union of each set $\pi_{1}\left(r_{i}\right)$ of each $r_{i}$.

\subsection{Analysis of Architectural Specifications}

Once translated to $\mathrm{mCRL} 2$, an architectural specification can be analysed and compared resorting to tools available in the corresponding toolsuit. The envisaged analysis is based on the following definition of architectural equivalence:

$$
a \equiv b \Leftrightarrow \operatorname{gen}_{h}(a) \approx_{B} \operatorname{gen}_{h}(b)
$$

where gen $_{h}$ refers to the same as gen in (14) but with each occurrence of conf replaced by conf $f_{h}$,i.e., the set of actions not involved in the interface are hidden; and $\approx_{B}$ denotes branching bisimilarity. Branching bisimilarity [4] relates behaviours differing in the amount of internal activity but exhibiting similar branching structure. This equivalence relation allows us to determine whether two architectures are interchangeable with respect to their interface behaviour.

A weaker form of comparing architectural specifications resorts to

$$
a \sqsubseteq b \Leftrightarrow \operatorname{gen}_{h}(a) \sqsubseteq_{W} \operatorname{gen}_{h}(b)
$$

where $\subseteq_{W}$ is weak trace inclusion. A weak trace is a sequence of observable actions performed by a behaviour. This relation allows us to answer if an architecture $b$ has an interface language allowing it to replace an architecture $a$.

Both $\approx_{B}$ and $\varsigma_{W}$ are supported by the mCRL2 toolset. In the sequel we illustrate the sort of envisaged analysis that can be carried out in our framework.

In order to start our comparisons we define a base architecture. We use the one in Listing 2 with the nested architecture $\mathrm{s}$ replaced with $\mathrm{s}=$ Server () an instance of the Server element defined in Listing 1.

Now suppose we want to charge the client each time the server is used. We define a new element PaidServer that after computing a response, calculates a cost for the service (Listing 7). Then we define the architecture paid by replacing $s$ with an instance of PaidServer, and we use the tool to check that both base $\subseteq$ paid and paid $\equiv$ base.

\section{Listing 7: Paid Server}

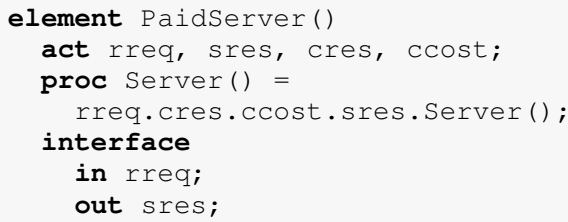

A new version of the server, represented by the element BuggyServer, is available. We define an architecture buggy by replacing $s$ with an instance of Buggyserver. Then we use the tool to establish that base ᄃ buggy but buggy $\equiv$ base. The second result is because while base responds to every request, buggy stops working in a non-deterministic way.

Listing 8: Buggy Server

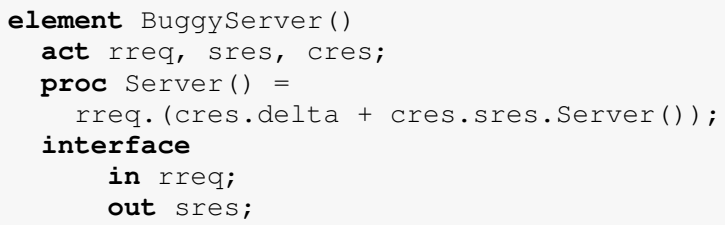

Now we want to compare base with an architecture in which the server does not perform any processing but can receive more than one request before responding. We name this architecture buffered, and we obtain it by replacing $\mathrm{s}$ in base with the $\mathrm{s}$ in Listing 9 . Now we verify that base 5 buffered but buffered $\neq$ base. This is because $\mathrm{s}$ in base has to respond to any received request before receiving a subsequent one, but the $\mathrm{s}$ in buffered can receive up to 3 requests without responding to any. 
Listing 9: Server with a 3-Position-Buffer

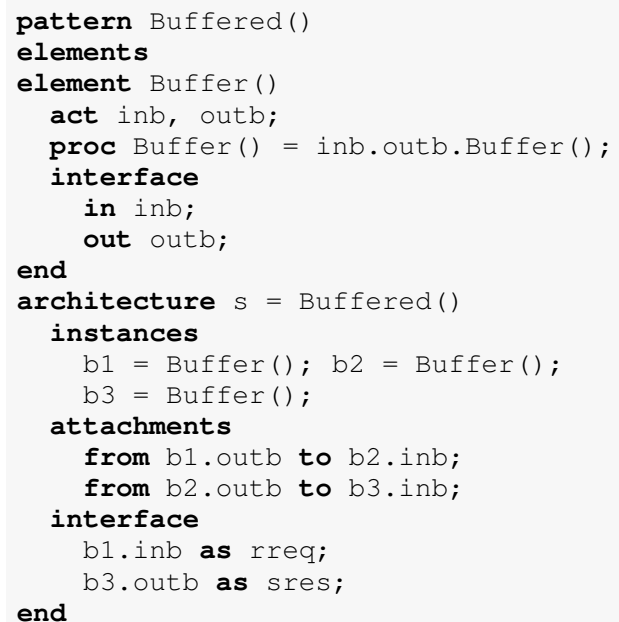

We define a different version of the server as a materialisation of the Pipe and Filter Pattern. It is the one shown in Listing 2 and we refer to such architecture as $p f$. Using the tool we are able to establish that base $\subseteq$ buffered $\subseteq p f$ and that $p f \equiv$ buffered $\equiv$ base. This means that the server in $p f$ can replace the server in base, and if we assume that the server in buffered is behaviourally correct, we can safely assume that the server in $p f$ also is.

We can add to the system the ability to acknowledge every communication. The architecture shown in Listing 10, named ackBase, reflects this behaviour. The server receives a request, computes a response, sends the response to the client, waits for an acknowledge indicating that the response was received, and then sends an acknowledge of receiving a request. As expected, base $\neq$ ackBase and base $\$$ ackBase.

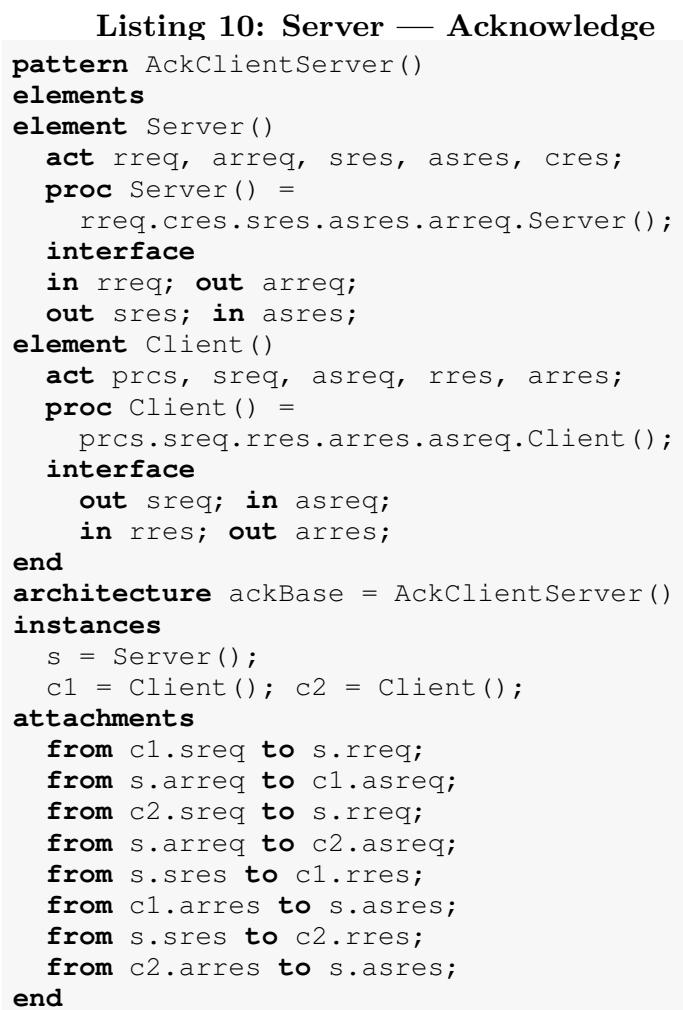


process algebra and in this way allow precise and analysable architectural descriptions. They also argue that this approach enhances usability of process algebras.

AADL, PADL and the language we propose in this paper have language constructs to specify components and configurations as first-class-citizens. In both PADL and the later it is also possible to explicitly declare connectors. Moreover, the same language construct is used for this.

Both PADL and AADL provide a variety of ports to indicate different sorts of interactions. In contrast, we only consider in and out ports, and resorts to the water-flow metaphor, to informally indicate their semantics.

The three languages allow the specification of types. Since AADL does not provide a language construct for connectors, it also lacks a mean to declare connector types.

The three languages support patterns as a mechanism to develop specifications from existing ones. PADL and our own language differ from AADL in that they particularly aim at formalising the concept of Architectural Pattern [6][15]. PADL provides a mechanism to restrict possible configurations that a system specified as an instance of a pattern may adopt. It basically limits how configurations can be built.

The three languages have specific tool support. Our language inherits the existing $\mathrm{mCRL} 2$ toolset, PADL is supported by a tool named Two-Towers, and AADL has an extensive tool support covering a wide range of needs.

\subsection{Conclusions and future work}

In this paper we presented an ADL that allows the behavioural modelling of architectural patterns and the compositional construction of architectures. We describe a translator from architectural specifications to mCRL2, enabling in this way, tool-supported analysis of the formers. The analysis considers two envisaged relations: equivalence and refinement; which are illustrated with examples. As future work we consider the development of a refinement calculus for architectural patterns, exploring relations $\equiv$ and $\sqsubseteq$ respectively defined in (26) and (27). We also expect to improve the translator in order to obtain process algebra specifications better suited for tool analysis.

\section{Acknowledgements}

This research was partially supported by Fct (the Portuguese Foundation for Science and Technology) under contract P TDC/EIA-CCO/108302/2008 - the MONDRIAN project, and by QREN (the Portuguese National Strategy Reference Chart) project 1621 - EvOLVE.

\section{REFERENCES}

[1] A. Aldini, M. Bernardo, and F. Corradini. A Process Algebraic Approach to Software Architecture Design, volume 54. Springer London, London, 2010.

[2] F. Arbab. Reo: a channel-based coordination model for component composition.
Mathematical Structures in Computer Science, 14(3):329-366, June 2004.

[3] J. Baeten. A brief history of process algebra. Theoretical Computer Science, 335(2/3):131-146, 2005.

[4] J. C. M. Baeten, T. Basten, and M. A. Reniers. Process Algebra: Equational Theories of Communicating Processes. Cambridge University Press, 2010

[5] J. P. Bodeveix, M. Filali, P. Gaufillet, and F. Vernadat. The AADL real-time model A behavioural annex for the AADL. In Proc. of the DASIA 2006 - DAta Systems In Aerospace Conference, number May, Berlin, Germany, 2006.

[6] Frank Buschmann, Regine Meunier, Hans Rohnert, Peter Sommerlad, and Michael Stal. Pattern-Oriented Software Architecture Volume 1: A System of Patterns. Wiley, 1996.

[7] D. Garlan. Style-based refinement for software architecture. Joint proceedings of the second international software architecture workshop (ISAW-2) and international workshop on multiple perspectives in software development (Viewpoints '96) on SIGSOFT '96 workshops -, pages 72-75, 1996.

[8] D. Garlan and M. Shaw. An introduction to software architecture. In V. Ambriola and G. Tortora, editors, Advances in Software Engineering and Knowledge Engineering (volume I). World Scientific Publishing Co., 1993.

[9] J. F. Groote, J. Keiren, A. Mathijssen, B. Ploeger, F. Stappers, C. Tankink, Y. Usenko, M. Weerdenburg, W. Wesselink, T. Willemse, and J. Wulp. The mcrl2 toolset. In Proc. International Workshop on Advanced Software Development Tools and Techniques (WASDeTT 2008), 2008.

[10] J. F. Groote, A. Mathijssen, M. Reniers, Y. Usenko, and M. van Weerdenburg. The formal specification language mcrl2. In Methods for Modelling Software Systems: Dagstuhl Seminar 06351, 2007.

[11] N. Medvidovic and R. N. Taylor. A classification and comparison framework for software architecture description languages. Software Engineering, IEEE, 26(1):70-93, 2002.

[12] R. Milner. Communication and Concurrency. Series in Computer Science. Prentice Hall, 1989.

[13] SAE Development Team. AADL homepage, 2011.

[14] A. Sanchez, L. S. Barbosa, and D. Riesco. A Language for Behavioral Modeling of Architectural Patterns Generation and Analysis in the mCRL2 Framework. Technical Report DI-CCTC-11-02, Universidade do Minho, Departamento de Informática, Campus de Gualtar 4710-057, Braga, Portugal, 032011.

[15] M. Shaw and D. Garlan. Software architecture: perspectives on an emerging discipline. Prentice Hall, 1996. 